\title{
Prevalence of Influenza A (H1N1) Seropositivity in Unvaccinated Healthcare Workers in Scotland at the Height of the Global Pandemic
}

\author{
Kate Smith, ${ }^{1}$ Pamela Warner, ${ }^{2}$ Linda J. Williams, ${ }^{2}$ Walt E. Adamson, ${ }^{3}$ \\ S. Vittal Katikireddi, ${ }^{1}$ Paul Dewart, ${ }^{4}$ William F. Carman, ${ }^{3}$ Kate Templeton, ${ }^{5}$ \\ Fiona C. Denison, ${ }^{6}$ and D. Graham Mackenzie ${ }^{1}$ \\ ${ }^{1}$ Public Health and Health Policy, NHS Lothian, Waverley Gate, 2-4 Waterloo Place, Edinburgh EH1 3EG, UK \\ ${ }^{2}$ Centre for Population Health Sciences, Medical School, University of Edinburgh, Teviot Place, Edinburgh EH8 9AG, UK \\ ${ }^{3}$ West of Scotland Specialist Virology Centre, Gartnavel General Hospital, Glasgow G12 0ZA, UK \\ ${ }^{4}$ St John's Hospital, Howden Road West, Livingston, West Lothian EH54 6PP, UK \\ ${ }^{5}$ Royal Infirmary Edinburgh, NHS Lothian, Edinburgh EH16 4SA, UK \\ ${ }^{6}$ MRC Centre for Reproductive Health, University of Edinburgh, Queen's Medical Research Institute, 47 Little France Crescent, \\ Edinburgh EH16 4TJ, UK
}

Correspondence should be addressed to D. Graham Mackenzie, graham.mackenzie@nhslothian.scot.nhs.uk

Received 31 May 2011; Revised 7 August 2011; Accepted 7 August 2011

Academic Editor: Ivo Iavicoli

Copyright (C) 2011 Kate Smith et al. This is an open access article distributed under the Creative Commons Attribution License, which permits unrestricted use, distribution, and reproduction in any medium, provided the original work is properly cited.

\begin{abstract}
Background. We set out to identify the level of previous exposure to influenza A (H1N1) in unvaccinated healthcare workers (HCWs) at the peak of the pandemic outbreak in the UK, with control samples collected prior to the outbreak. Methods. Crosssectional study (seroprevalence assessed before and at pandemic peak, with questionnaire data collected at peak of outbreak) in HCWs in Scotland. Results. The prevalence of seropositivity in $493 \mathrm{HCWs}$ at pandemic peak was $10.3 \%$, which was higher than the prepandemic level by 3.7 percentage points ( $95 \%$ CI $0.3 \%$ to $7.3 \%, P=0.048$ ). Seropositivity rates for frontline and nonfrontline HCWs were similar. Conclusion. At pandemic peak, only 10.3\% of HCWs were seropositive for influenza A (H1N1), so the great majority were still susceptible to infection at the introduction of the vaccination programme. Few studies have reported on seroprevalence in unvaccinated and asymptomatic participants, so our findings may have relevance to the wider population.
\end{abstract}

\section{Introduction}

The susceptibility of healthcare workers (HCW) to influenza is relevant in terms of sickness absence, productivity, and onward transmission of infection from carer to patient [1], a particular issue with a novel influenza strain. The first cases of 2009 pandemic influenza A (H1N1) were identified in Scotland in April 2009 [2]. We set out to determine the seroprevalence of antibodies against the virus in unvaccinated HCWs in Lothian, south east Scotland in Autumn 2009 (after the first wave of infection, but before the expected increase in cases over the winter period). It is now clear that this was close to the peak of the outbreak in the United Kingdom (UK) [3].

\section{Materials and Methods}

Between 29/10/2009 and 4/11/2009 (at the time believed to be midpandemic, but now known to have been peak), unvaccinated NHS Lothian employees $(n=505)$ were recruited within days of the start of the HCW vaccination programme and prior to most HCWs being vaccinated. Recruitment was mainly from three acute teaching hospitals, with smaller numbers from a psychiatric hospital and the Health Board headquarters. After giving informed consent participants had a serum sample taken and completed a short questionnaire recording sex, age, occupation, and selfreported history of flu-like symptoms or illness since the start of the pandemic period (April 2009). Of the 505 recruited, 
493 employees were aged between 16 and 65 years and had complete information (laboratory results and questionnaire response). It is these 493 respondents who have been used in all analyses reported here. Serology specimens were tested for antibodies to influenza A (H1N1) in the West of Scotland Specialist Virology Centre, Glasgow, using microneutralisation assays at a dilution of 1:40 as previously described [4]. In addition a set of age- and sex-matched blood samples ( $n=471$ ) were obtained from stored serology specimens collected from HCWs for occupational health purposes during 2008 (i.e., prepandemic), and these were also tested. Chi-squared test with Yates' continuity correction was used to compare seropositive prevalence in pre- and peak pandemic samples and logistic regression analysis to examine the association of seropositivity with risk group classification. The study was approved by the Local Regional Ethics Committee. Sample size needed was calculated as 500, to ensure a pandemic estimate of seropositivity with $95 \%$ confidence interval no wider than \pm 3.5 percentage points, assuming that the observed prevalence was $20 \%$.

\section{Results}

The age and sex profile for pre- and peak pandemic samples was similar to the overall hospital-based NHS Lothian workforce, and $67 \%$ of pandemic participants were classified as frontline, the same as the overall HCW workforce.

In the pandemic sample the prevalence of seropositivity in HCWs was $10.3 \%$ (95\% confidence interval (CI) 7.7 to $13.0 \%)$. This seropositivity was higher than prepandemic HCW seropositivity rate by 3.7 percentage points (95\% CI 0.3 to 7.3 percentage points, $P=0.048$ ). The study was not powered to test for variation in seropositivity rates across age bands, but the highest pandemic rate observed was $17.4 \%$ in the youngest age band (16 to 25 years old), whereas prepandemic this age band had nearly the lowest rate (4.3\%). However, the small numbers in the youngest age band means that the difference in seropositivity (pre versus pandemic) was not statistically significantly different according to whether comparing within those aged up to 25 years or over 25 years $(P=0.147)$.

Occupations were grouped into frontline contact (allied health professionals, doctors, nurses, midwives, and students) and non-frontline contact (administrative, pharmacy, and support staff including e.g., individuals working in laboratories or "estates"). For pandemic samples, seropositivity rates for frontline and non-frontline HCWs were similar overall at $11.0 \%$ (95\% CI 7.6 to $14.4 \%$ ) and $9.1 \%$ (5.8 to $12.5 \%$ ), respectively. Influenza-like symptoms in the preceding six months were reported by 208 (42.2\%), and $12.0 \%$ (95\% CI 7.6 to $16.4 \%$ ) of them were seropositive, compared to $9.1 \%(95 \%$ CI 5.8 to $12.5 \%)$ of those without recent symptoms (Table 1).

A trend analysis across three levels of risk (prepandemic, pandemic but no influenza-like symptoms, pandemic reporting symptoms) found a statistically significant trend in seropositivity $(P=0.018)$, with a linear odds ratio of 1.39 (95\% CI 1.06 to 1.84 ), suggesting that overall the odds of a

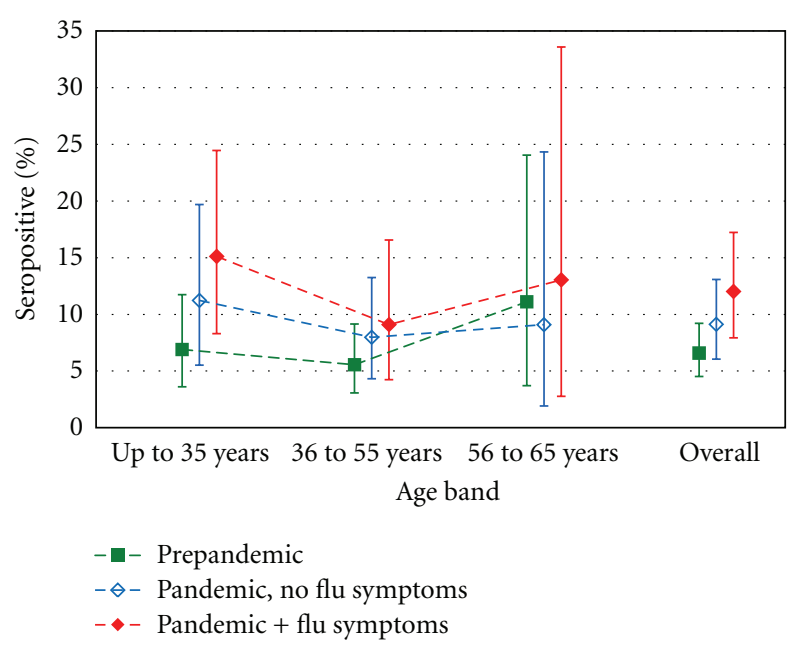

FIGURE 1: Seropositivity separately for peakpandemic and prepandemic samples, with peakpandemic samples subdivided according to report or not of influenza-like symptoms $(n=208,285,471$, resp.), overall and by age band.

positive laboratory result increased by $39 \%$ for each move from one risk category to the next higher category. Figure 1 shows overall seropositivity for the three risk categories and across age bands.

\section{Discussion}

To our knowledge, this is the first study in the UK to quantify the level of seropositivity to influenza A (H1N1) in unvaccinated HCWs pre- and peak pandemic. Our findings have important implications both for understanding the spread of influenza $\mathrm{A}(\mathrm{H} 1 \mathrm{~N} 1)$ and for planning and delivery of future pandemic influenza vaccination programmes.

Previous vaccination is indistinguishable from previous infection on microneutralisation testing, so our active recruitment of unvaccinated participants for the pandemic samples avoids the limitation of other studies which did not have full documentation of vaccination status, many of which relied on discarded laboratory samples, samples from blood donors or patients [4-7]. Other studies have looked at a single time point [4], including one study of HCWs (from Taiwan) [8], which means that it is not possible to compare prepandemic immunity observed in other studies [6].

Our study included pre- and pandemic samples which allowed us to compare peak pandemic seropositivity against the rate about 9 months before the start of the pandemic. The only other HCW study conducted pre- and midpandemic that we have identified (from Singapore) used a different assessment of seroconversion, requiring a 4-fold rise in titre from baseline, so a direct comparison with our findings is not possible [9].

Elder has reported previously that there is little indication of increased susceptibility to seasonal influenza by occupational group, including healthcare [1]. Accordingly, in the absence of a more general study of seroprevalence in unvaccinated adults of working age, our estimate of 


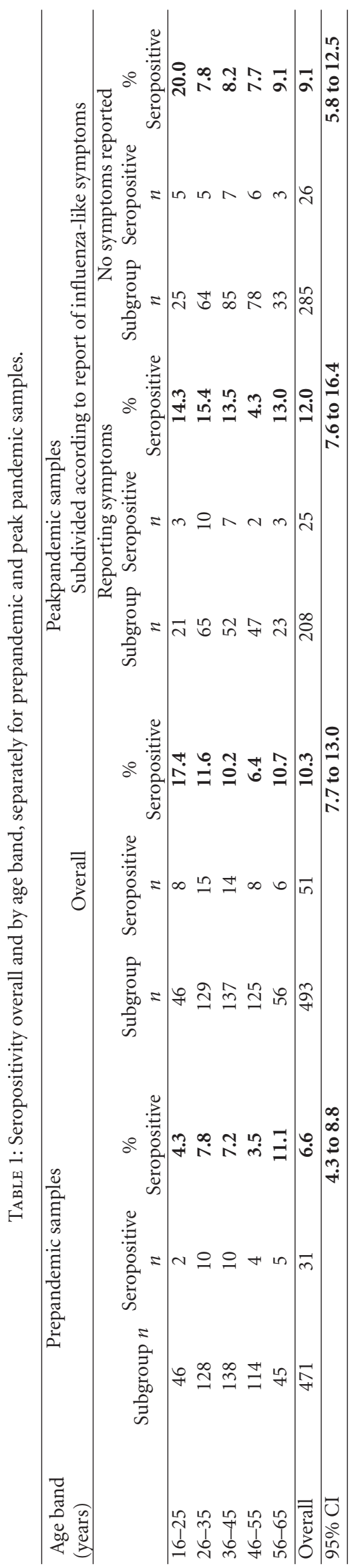


pandemic seropositivity is arguably the closest estimate to date of overall population prevalence in adults in the UK at that time.

It is important to remember the levels of uncertainty and concern that existed midpandemic. In October 2009 the Scottish Chief Medical Officer noted a slower spread than anticipated [10]. However that same week the United States declared an influenza A (H1N1) emergency, with 1,000 deaths across 46 states and questions about the ability to cope with a potential surge in cases [11]. A week later Health Protection Scotland reported that influenza A (H1N1) related hospitalisations and deaths in Scotland continued to increase [12]. Furthermore, the influenza virus's ability to mutate and transfer genetic material between strains meant that a substantial increase in cases over the winter influenza season remained a possibility. Our findings show that over the first six months of the pandemic the A (H1N1) strain was of limited virulence with only around $4 \%$ of susceptible HCWs having developed seropositivity despite $42 \%$ participants reporting flu-like symptoms in preceding months. However, previous virulence is not necessarily a guide to future spread, particularly on the cusp of the influenza season, and our findings show that $90 \%$ HCWs were still susceptible to infection. These findings support the importance of vaccination even at the peak of a pandemic (and regardless of previous symptoms), contrasting with findings of a large English study that sampled patients accessing health care between August and September 2009 [6].

It has been estimated that between 40 and $50 \%$ of HCWs in the UK were vaccinated for influenza A (H1N1) by February-April 2010 [13-15]; in Lothian the figure was 52\% [16]. This high level of uptake in HCWs across the United Kingdom in the weeks following this study means that it would not be feasible to estimate postpandemic seropositivity among unvaccinated HCWs. Although in other studies performed towards the end of the pandemic period the seroprevalence reported-between 20 and $40 \%[4,5,7]-$ was considerably higher than we found, there is uncertainty about vaccination rates and caveats about study population in these studies, as described above.

Our study has a number of potential weaknesses. We did not recruit primary care staff. Self-selection of participants and the exclusion of HCWs who were targeted first by the vaccination programme may have led to an under- or overestimation of the true level of infection with the virus for all HCWs in Lothian. Other studies have demonstrated a greater increase in seropositivity during the pandemic period in younger adults ( 16 to 25 years old) compared to older adults, both in high prevalence areas in the UK [4] and among blood donors in Australia [5]. While our study showed a similar pattern of greater increase in seropositivity in younger adults than other age groups (Figure 1) it was not powered to test such an interaction hypothesis.

These findings have important implications for research into future pandemics. Having information on seroprevalence in unvaccinated individuals during the pandemic would have been invaluable and may be feasible in a future pandemic. Virology samples can be stored indefinitely allowing comparison with samples taken from previous years.
Such information would help identify susceptible age groups, helping the planning of vaccination campaigns during the pandemic. We therefore suggest that health protection organisations consider collecting samples annually from a representative "panel" of asymptomatic individuals, selected and powered to allow comparisons by age.

\section{Conclusion}

In conclusion, our study shows that at pandemic peak $10.3 \%$ of HCWs in Lothian, south east Scotland, were seropositive for influenza A (H1N1), so the great majority were still susceptible to influenza A (H1N1) infection at the introduction of the vaccination programme.

\section{Conflict of Interests}

The authors declare that there is no conflict of interests.

\section{Ethical Approval}

This study was approved by the Lothian LREC (reference no. 09/S1102/44).

\section{Acknowledgments}

Dr. Kirsty Dundas (Consultant in Obstetrics and Gynaecology, Royal Infirmary Edinburgh, NHS Lothian), Eilidh Fletcher (Analyst, Health Intelligence Unit, NHS Lothian), Andrew Deas and Mairi McCulloch (Information Services Division, NHS National Services Scotland). Sharon Cameron and colleagues at the Wellcome Trust Clinical Research Facility, Royal Infirmary Edinburgh. The Chief Scientist Office, Scottish Government Health Directorates, for funding this work and providing support throughout the research.

\section{References}

[1] A. G. Elder, "Influenza in working populations: an overview," Occupational Medicine, vol. 52, no. 5, pp. 239-240, 2002.

[2] E. McLean, R. G. Pebody, C. Campbell et al., "Pandemic (H1N1) 2009 influenza in the UK: clinical and epidemiological findings from the first few hundred (FF100) cases," Epidemiology and Infection, vol. 138, no. 11, pp. 1531-1541, 2010.

[3] "Epidemiological report of pandemic (H1N1) 2009 in the UK April 2009-May 2010," Health Protection Agency, 2010, http://www.hpa.org.uk/web/HPAwebFile/HPAweb_C/ 1284475321350.

[4] W. E. Adamson, S. Maddi, C. Robertson et al., "2009 pandemic influenza $\mathrm{A}(\mathrm{H} 1 \mathrm{~N} 1)$ virus in Scotland: geographically variable immunity in Spring 2010, following the winter outbreak," Eurosurveillance, vol. 15, no. 24, 2010.

[5] J. Mcvernon, K. Laurie, T. Nolan et al., "Seroprevalence of 2009 pandemic influenza a (H1N1) virus in Australian blood donors, October-December 2009," Eurosurveillance, vol. 15, no. 40 , pp. 7-14, 2010.

[6] E. Miller, K. Hoschler, P. Hardelid, E. Stanford, N. Andrews, and M. Zambon, "Incidence of 2009 pandemic influenza A H1N1 infection in England: a cross-sectional serological study," The Lancet, vol. 375, no. 9720, pp. 1100-1108, 2010. 
[7] S. M. Zimmer, C. J. Crevar, D. M. Carter et al., "Seroprevalence following the second wave of pandemic $2009 \mathrm{H} 1 \mathrm{~N} 1$ influenza in Pittsburgh, PA, USA," PLoS One, vol. 5, no. 7, Article ID e11601, 2010.

[8] Y. J. Chan, C. L. Lee, S. J. Hwang et al., "Seroprevalence of antibodies to pandemic (H1N1) 2009 influenza virus among hospital staff in a medical center in Taiwan," Journal of the Chinese Medical Association, vol. 73, no. 2, pp. 62-66, 2010.

[9] M. I. C. Chen, V. J. M. Lee, W. Y. Lim et al., "2009 influenza $\mathrm{A}(\mathrm{H} 1 \mathrm{~N} 1)$ seroconversion rates and risk factors among distinct adult cohorts in Singapore," Journal of the American Medical Association, vol. 303, no. 14, pp. 1383-1391, 2010.

[10] Chief Medical Officer Scotland, "Launch date for the influenza $\mathrm{A}(\mathrm{H} 1 \mathrm{~N} 1)$ vaccination programme $2009-2010$ and additional details on vaccine dosage," CMO Letter 12, Scottish Government Health Directorate, 2009, http://www.sehd.scot.nhs .uk/cmo/CMO(2009)12.pdf.

[11] BBC News, " US declares swine flu 'emergency'” BBC News Report, 2009, http://news.bbc.co.uk/1/hi/8324070.stm.

[12] "Weekly influenza situation report (Including H1N1v)," Health Protection Scotland, 2010, http://www.documents.hps .scot.nhs.uk/respiratory/swine-influenza/situation-reports/ weekly-influenza-sitrep-2009-10-29.pdf.

[13] "Pandemic H1N1(Swine Flu) and seasonal influenza vaccine uptake amongst frontline healthcare workers in England 2009/2010,"Department of Health, 2010, http://www.dh.gov .uk/en/Publicationsandstatistics/Publications/PublicationsPolicyAndGuidance/DH_121011.

[14] "Influenza A H1N1v," Situation Report number 72, Scottish Government Resilience Room, 2010.

[15] "NHS Lanarkshire Director of Public Health Annual report 2009/2010," (chapter 3.1, Influenza A (H1N1v) in Lanarkshire), NHS Lanarkshire, 2010, http://www.nhslanarkshire.org.uk/Services/PublicHealth/directors-annual-report2009-10/Documents/Health\%20Protection.pdf.

[16] E. Fletcher, "Informational analyst," NHS Lothian, Personal communication, 2011. 


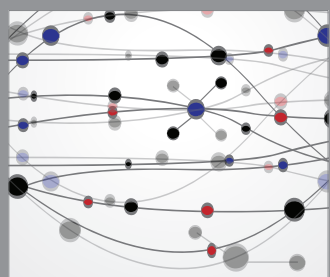

The Scientific World Journal
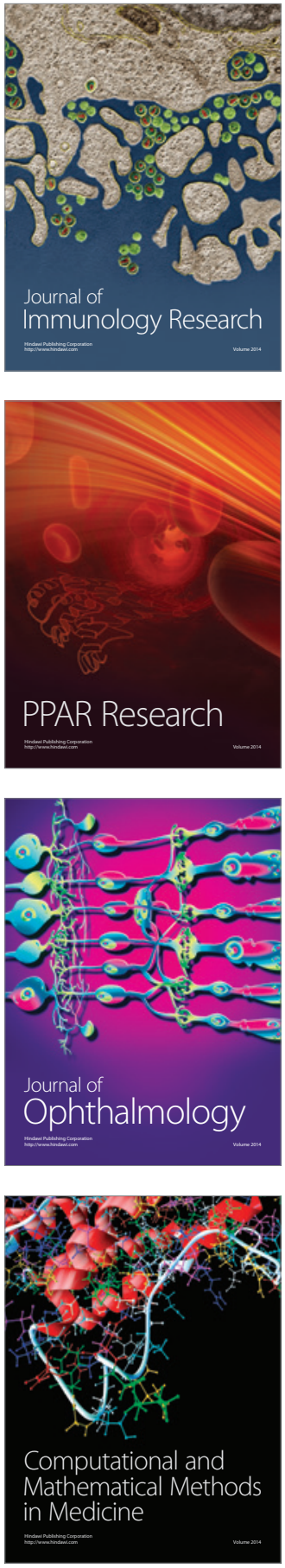

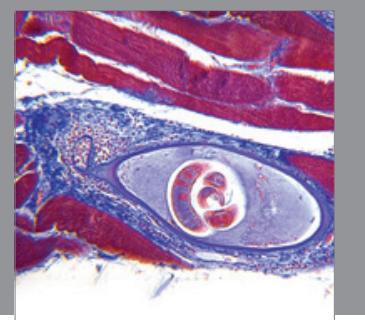

Gastroenterology

Research and Practice
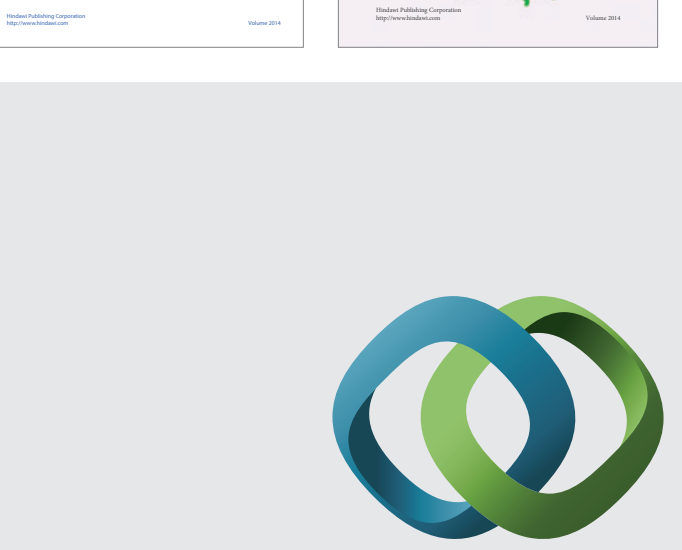

\section{Hindawi}

Submit your manuscripts at

http://www.hindawi.com
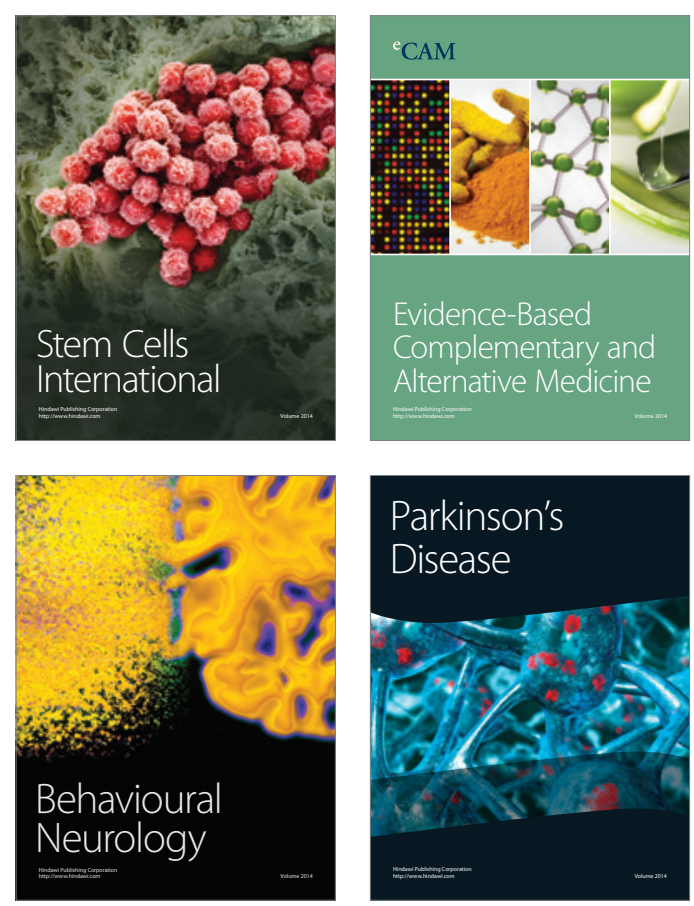

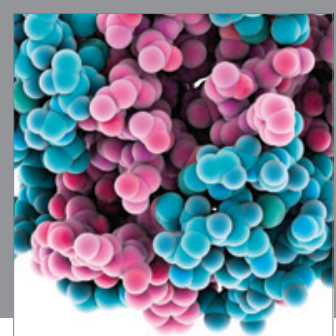

Journal of
Diabetes Research

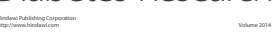

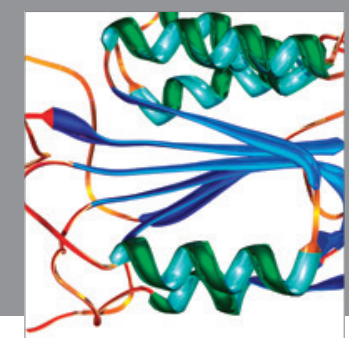

Disease Markers
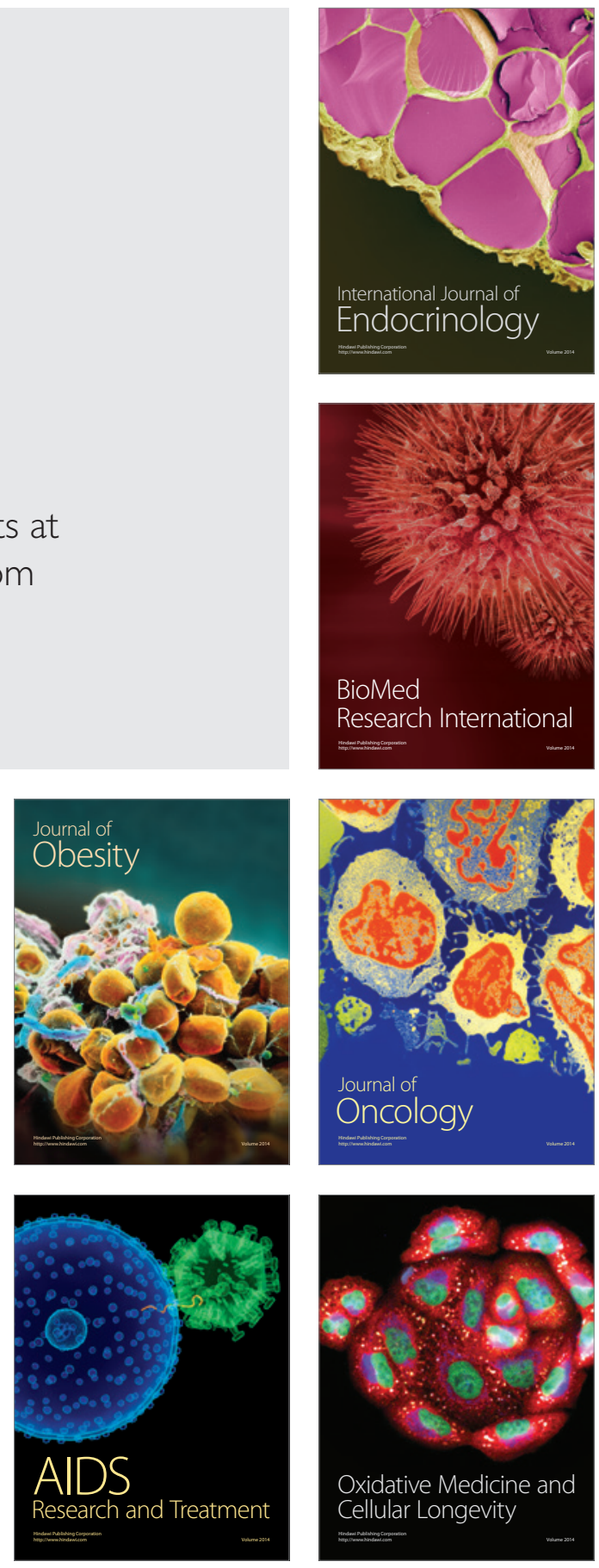